\title{
Uso do fentanil transdérmico para analgesia pós-operatória em cirurgia de artrodese de coluna*
}

\author{
Percutaneous fentanyl for postoperative spinal arthrodesis analgesia
}

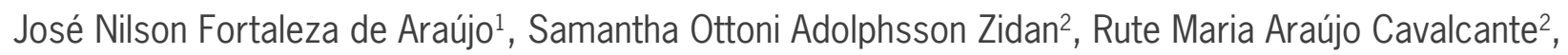
Roberto Cesar Ponte Ibiapina ${ }^{1}$

* Recebido do Instituto Doutor José Frota. Fortaleza. CE

\section{RESUMO}

JUSTIFICATIVA E OBJETIVOS: O controle da dor pós-operatória de moderada a intensa ainda é um problema na prática diária devido à dor nos intervalos entre a administração de doses de analgésicos e os efeitos adversos. O fentanil transdérmico apresenta analgesia constante por até 72 horas, tem sido usado para analgesia na dor aguda. O objetivo deste estudo foi avaliar a analgesia proporcionada pelo uso do fentanil transdérmico na dor aguda pós-operatória em pacientes submetidos à cirurgia de artrodese de coluna, bem como observar a incidência de efeitos adversos relacionados ao seu uso.

MÉTODO: Foram incluídos 9 pacientes submetidos à artrodese de coluna sob anestesia geral com propofol, sufentanil e cisatracúrio e manutenção com remifentanil e isoflurano. Um adesivo de fentanil transdérmico de 12 $\mu \mathrm{g} / \mathrm{h}$ foi aplicado antes da cirurgia e ao término da cirurgia, cetoprofeno, dipirona e tramadol foram administrados. Dipirona foi aplicada cada $6 \mathrm{~h}$ como analgésico de resgate. A intensidade da dor foi avaliada a cada 12 $\mathrm{h}$ pela escala analógica visual e o consumo de dipirona e cetoprofeno em intervalos $12 \mathrm{~h}$ e os efeitos adversos foram anotados.

1. Médico Anestesiologista do Instituto Doutor José Frota. Fortaleza. CE, Brasil.

2. Médica Residente de Anestesia do Instituto Doutor José Frota. Fortaleza. CE, Brasil.

Endereço para correspondência:

Dr. José Nilson Fortaleza de Araújo

Rua Senador Pompeu, 1757 - Centro

60025-001 Fortaleza, CE.

Fone (85) 3255-5000

E-mail: nilsonfortaleza@gmail.com
RESULTADOS: Após $12 \mathrm{~h}$ da aplicação do adesivo, a média da intensidade da dor era de $2,11 \pm 2,84$. O maior valor da intensidade da dor foi $60 \mathrm{~h}$ após a aplicação do adesivo e os menores valores foram constatados entre 24 e $72 \mathrm{~h}$ depois da aplicação do adesivo de fentanil transdérmico. O maior consumo total de dipirona foi de 8000 $\mathrm{mg}$ no período compreendido entre 12 e $24 \mathrm{~h}$ e entre 36 e $48 \mathrm{~h}$ e o menor consumo foi de zero mg no período compreendido entre 60 e $72 \mathrm{~h}$, porém não houve diferença estatisticamente significativa nos intervalos observados $(p=0,47)$. Os efeitos colaterais foram um caso de sonolência e outro de náuseas e vômitos.

CONCLUSÃO: Os resultados deste estudo sugerem que o fentanil transdérmico mostrou-se seguro e efetivo para analgesia pós-operatória de cirurgia de coluna. Os resultados também evidenciaram que o fentanil transdérmico apresentou efeitos colaterais pouco relevantes.

Descritores: Adesivo transdérmico, Analgesia, Artrodese, Coluna vertebral, Fentanil.

\section{SUMMARY}

BACKGROUND AND OBJECTIVES: Moderate to severe postoperative pain control is still a daily problem due to pain in the intervals between analgesic administration and adverse effects. Percutaneous fentanyl induces continuous analgesia for up to 72 hours and has been used to control acute pain. This study aimed at evaluating percutaneous fentanyl analgesia to control acute postoperative pain in patients submitted to spinal arthrodesis, as well as at observing the incidence of fentanyl-related adverse effects.

METHOD: Participated in this study 9 patients submitted to spinal arthrodesis under general anesthesia with propofol, sufentanil and cisatracurium and maintenance 
with remifentanil and isoflurane. One $12 \mu \mathrm{g} / \mathrm{h}$ percutaneous fentanyl adhesive was applied before surgery and ketoprofen, dipirone and tramadol were administered at surgery completion. Dipirone was administered every 6 hours as rescue analgesic drug. Pain intensity was evaluated every 12 hours by the visual analog scale and dipirone and ketoprofen consumption in 12-hour intervals; adverse effects were recorded.

RESULTS: Twelve hours after adhesive application, mean pain intensity was $2.11 \pm 2.84$. Highest pain intensity was observed 60 hours after adhesive application and lowest value was observed 24 and 72 hours after percutaneous fentanyl adhesive application. Highest total dipirone consumption was $8000 \mathrm{mg}$ in the period between 60 and 72 hours and between 36 and 48 hours and the lowest consumption was zero $\mathrm{mg}$ in the period between 60 and 72 hours, but there has been no statistically significant difference in observed periods ( $\mathrm{p}=$ 0.47). Side effects were one case of sleepiness and one of nausea and vomiting.

CONCLUSION: This study results suggest that percutaneous fentanyl is safe and effective for postoperative spinal surgery pain. Results have also shown that percutaneous fentanyl has minor side effects.

Keywords: Analgesia, Arthrodesis, Fentanyl, Percutaneous adhesive, Spine.

\section{INTRODUÇÃO}

O controle da dor pós-operatória de moderada a intensa ainda é um problema constante na prática diária ${ }^{1}$ e está relacionada com o aumento de complicações pulmonares e tromboembólicas no pós-operatório, aumento do tempo de internação, piora na qualidade de vida do paciente, além de aumento no risco de desenvolvimento de dor crônica ${ }^{2}$.

Um dos fatores que contribuem para a inadequada analgesia é a ocorrência de dor nos intervalos entre a administração de doses de analgésicos e os efeitos adversos ${ }^{3}$. $\mathrm{O}$ fentanil administrado por via transdérmica, que apresenta farmacocinética similar ao uso por via venosa com a concentração plasmática se mantendo constante durante o período de até 72 horas $^{4}$, tem se mostrado um método seguro e efetivo no manuseio da dor crônica, e também tem sido usado para promoção de analgesia também na dor aguda ${ }^{5,6}$.

O objetivo deste estudo foi avaliar a analgesia proporcionada pelo uso do fentanil transdérmico na dor aguda pós-operatória em pacientes submetidos à cirurgia de artrodese de coluna, bem como observar a incidência de efeitos adversos relacionados ao seu uso.

\section{MÉTODO}

Após a aprovação pelo Conselho de Ética em Pesquisa da Instituição (protocolo 510/2011), realizou-se este estudo prospectivo sendo incluídos 9 pacientes submetidos à cirurgia de artrodese de coluna que, concordaram em participar da pesquisa mediante assinatura de Termo de Consentimento Livre e Esclarecido. Os critérios de inclusão foram idade maior que 18 anos e menor que 60 anos, estado físico ASA I e II, estar no pós-operatório desperto, alerta e respirando espontaneamente com saturação de oxigênio maior ou igual a $90 \%$ sem o uso de oxigênio suplementar. Foram excluídos pacientes com diabetes, déficits neurológicos sensitivos e de linguagem, doenças pulmonares, cardíacas, etilistas, em uso de fármacos depressores do sistema nervoso central, dependentes de opioides ou em uso de analgesia regional contínua.

Antes da administração da medicação pré-anestésica os pacientes foram esclarecidos sobre a finalidade do adesivo e orientados a não retirá-lo antes das $72 \mathrm{~h}$ de uso. Um adesivo de fentanil transdérmico com de $12 \mu \mathrm{g} / \mathrm{h}$ foi aplicado na pele limpa e seca da região interescapular 30 minutos antes do início da cirurgia. Midazolam na dose de $0,05 \mathrm{mg} / \mathrm{kg}$ foi usado como medicação pré-anestésica, e a anestesia foi induzida com $2 \mathrm{mg} / \mathrm{kg}$ de propofol, $0,5 \mu \mathrm{g} / \mathrm{kg}$ de sufentanil e $0,1 \mathrm{mg} / \mathrm{kg}$ de cisatracúrio e mantida com $0,2-0,5 \mu \mathrm{g} / \mathrm{kg} / \mathrm{min}$ de remifentanil e isoflurano em vaporizador calibrado. Após o término da anestesia os pacientes foram transportados até a sala de recuperação pós-anestésica (SRPA), aonde foram mantidos sob observação por 24 horas.

Como existe um retardo de 6 a 12 horas para o platô do efeito analgésico do fentanil transdérmico, foi administrado, por via venosa, 6 horas após o término da cirurgia dose única de $100 \mathrm{mg}$ de tramadol e $30 \mathrm{mg} / \mathrm{kg}$ de dipirona. Como analgésicos de resgate foram prescritos 30 $\mathrm{mg} / \mathrm{kg}$ de dipirona a cada 6 horas e $100 \mathrm{mg}$ cetoprofeno de acordo com a necessidade. Não foram prescritos fármacos depressores do SNC, tais como antidepressivos ou ansiolíticos.

Os pacientes foram acompanhados durante 3 dias e a intensidade da dor foi avaliada pela escala analógica visual (EAV) a cada 12 horas, e a quantidade de dipirona e cetoprofeno usados com analgésico de resgate foram avaliados em períodos de 12 horas. Foram anotados os efeitos adversos a cada 12 horas.

Os dados foram submetidos à análise estatística pela Análise de Variância (ANOVA), e os resultados foram expressos em média e desvio-padrão. O nível de significância considerado foi 0,05 . 


\section{RESULTADOS}

Foram incluídos no estudo 7 pacientes estado físico ASA I e 2 pacientes ASA II, sendo 6 homens e 3 mulheres com média de idade de $42,33 \pm 10,92$ anos e de peso de $64,11 \pm 7,68 \mathrm{~kg}$.

Após $12 \mathrm{~h}$ da aplicação do adesivo, a média da intensidade da dor era de $2,11 \pm 2,84$. O maior valor da intensidade da dor foi $60 \mathrm{~h}$ após a aplicação do adesivo e os menores valores foram constatados 24 e $72 \mathrm{~h}$ depois da aplicação do adesivo de fentanil transdérmico (Tabela 1).

Tabela 1 - Intensidade da dor avaliada pela escala analógica, expressa em média \pm DP

\section{Momentos da Avaliação}

Intensidade da Dor da Dor (horas)

\begin{tabular}{ll}
\hline 12 & $2,11 \pm 2,84$ \\
24 & $1,44 \pm 1,87$ \\
36 & $2,00 \pm 2,17$ \\
48 & $2,22 \pm 1,71$ \\
60 & $2,31 \pm 2,23$ \\
72 & $1,44 \pm 1,87$ \\
\hline
\end{tabular}

O maior consumo de total dipirona foi de $8000 \mathrm{mg}$ no período compreendido entre 12 e $24 \mathrm{~h}$ e entre 36 e $48 \mathrm{~h}$ e o menor consumo foi de zero mg no período compreendido entre 60 e $72 \mathrm{~h}$, porém não houve diferença estatisticamente significativa nos intervalos observados $(\mathrm{p}=$ 0,47) (Tabela 2).

Nenhum dos pacientes observados apresentou prurido, retenção urinária, reação no local da aposição do adesivo ou depressão respiratória. Um dos pacientes referiu sonolência sem consequência clínica. Outro apresentou náusea seguida de vômito, que cedeu depois do tratamento com metoclopramida. Nenhum adesivo foi retirado inadvertidamente durante o período avaliado.

Tabela 2 - Consumo de dipirona em miligramas, nos vários períodos de 12 horas.

\begin{tabular}{ccc}
\hline Períodos & \multicolumn{2}{c}{ Consumo de Dipirona } \\
& Total & Média \pm DP \\
\hline $12-24 \mathrm{~h}$ & 8000 & $880 \pm 1050$ \\
$24-36 \mathrm{~h}$ & 4000 & $440 \pm 880$ \\
$36-48 \mathrm{~h}$ & 8000 & $880 \pm 2020$ \\
$48-60 \mathrm{~h}$ & 6000 & $660 \pm 1000$ \\
$60-72 \mathrm{~h}$ & 0 & 0 \\
\hline
\end{tabular}

\section{DISCUSSÃO}

O desenvolvimento de métodos mais efetivos para o alívio da dor pós-operatória é uma questão de fundamental importância. Não apenas a dor relacionada ao trauma operatório agudo é fonte de imenso sofrimento, mas também está relacionada com dor crônica debilitante e estresse pós-traumático ${ }^{7}$. Diversos estudos mostram que, apesar do esforço para melhorar o controle da dor pós-operatória com o desenvolvimento e implementação de guidelines, o manuseio da dor ainda é dificil ${ }^{8,9}$. Estudo evidenciou proporção significativa de pacientes recebendo analgesia pós-operatória que ainda referiam dor de intensidade moderada à intensa ${ }^{10}$.

Os pacientes podem experimentar dor, enquanto aguardam a equipe de enfermagem administrar doses de opioides em bolus, ou durante a transição entre duas modalidades de analgesia ${ }^{3}$.

$\mathrm{O}$ ajuste da analgesia após procedimentos cirúrgicos não consiste apenas no tratamento da dor, pois também se faz necessário o controle dos efeitos adversos, de forma que estes estejam ausentes ou possam ser contornados sem lesão ao paciente.

A analgesia com o fentanil transdérmico é feita sem o uso de agulhas e apresenta farmacocinética similar ao uso do fármaco por via venosa, e a concentração plasmática de fentanil se mantém constante durante o período de aplicação do adesivo, por até 72 horas ${ }^{4}$. Estudos comparando ambas as apresentações, sob o ponto de vista farmacocinético, demonstraram meia-vida e concentração máxima semelhante entre a infusão contínua e o adesivo $^{11}$.

A natureza não invasiva do método minimiza as complicações relacionadas à agulha e a punções venosas, além de também eliminar o risco de intervalos na analgesia, o que é inerente aos métodos não contínuos ${ }^{12}$. Uma preocupação importante relativa à analgesia aguda com opioides é a possibilidade de insuficiência respiratória, entretanto estudos comprovam a segurança do fentanil transdérmico para esses pacientes ${ }^{6,13-15}$.

Estudo demonstrou que o uso de fentanil por via transdérmica proporcionou analgesia satisfatória com baixas intensidades de dor. Ao comparar valores obtidos pela EAV nos intervalos observados, foram obtidas médias que oscilaram entre 1,44 e 2,31, o que caracteriza, de acordo com a escala numérica ${ }^{16}$, dor de leve intensidade. Procedimentos cirúrgicos como artrodese de coluna são sabidamente acompanhados de grande potencial doloroso, e o fentanil transdérmico mostrou ser um grande aliado na analgesia desses pacientes. 
O fentanil transdérmico já teve sua eficácia comprovada, semelhante à morfina através de analgesia controlada pelo paciente (ACP) por alguns autores ${ }^{6,17}$. Algumas vantagens são a praticidade de administração do adesivo, a não dependência de acesso venoso ou de pessoal treinado em bombas de analgesia. Soma-se a isso a maior comodidade e liberdade de deambulação, proporcionando maior aceitação por parte do paciente e de toda equipe hospitalar ${ }^{18}$. A morfina administrada por ACP, por sua complexidade, possui um potencial de apresentar diversos problemas como erros de programação, mau funcionamento do sistema venoso por desconexão, flebites, obstruções, bolus acidental, dentre outros, que podem levar à ocorrência de subdoses ou sobredoses de opioide ${ }^{19}$. Devido ao fato do fentanil ser administrado por via transdérmica e não necessitar de programação, complicações relacionadas ao uso são menos frequentes. É sabido que o início de ação obtido ocorre aproximadamente após $12 \mathrm{~h}$ de uso, portanto é necessário propiciar analgesia potente para as primeiras $12 \mathrm{~h}$, bem como aplicar o adesivo precocemente para abreviar este período.

A ocorrência de efeitos adversos em estudo utilizando fentanil transdérmico iontoforético, quando comparado ao uso de morfina por ACP, foi semelhante no contexto geral, incluindo eventos graves como depressão respiratória. A incidência de prurido foi maior no grupo da morfina e estatisticamente significante. $\mathrm{O}$ efeito adverso hipotensão foi mais frequente no grupo da morfina, mas sem significância estatística $(\mathrm{p}=0,06)^{6}$.

No presente estudo, utilizando fentanil transdérmico na dose de $12 \mu \mathrm{g} / \mathrm{h}$ pode-se observar baixa incidência de efeitos adversos. Não foram encontrados efeitos adversos, tais como prurido, retenção urinária e depressão respiratória. A ocorrência de náuseas e vômitos em apenas um paciente e a incidência de sonolência em outro do total de 9 pacientes avaliado, reforça a segurança dessa forma de apresentação do opioide. Tais efeitos foram pouco relevantes, infrequentes e facilmente controlados. São necessários outros estudos com maior número de pacientes para avaliar de modo mais amplo a segurança e eficácia desta apresentação do fentanil em analgesia pós-operatória, de cirurgias que apresentam dor intensa no pós-operatório imediato.

\section{CONCLUSÃO}

Os resultados deste estudo sugerem que o fentanil transdérmico de $12 \mu \mathrm{g} / \mathrm{h}$ mostrou-se seguro e efetivo para analgesia pós-operatória de cirurgia de coluna, com efeitos colaterais pouco relevantes.

\section{REFERÊNCIAS}

1. Mattia C, Coluzzi F. Acute postoperative pain management: focus on iontophoretic transdermal fentanyl. Ther Clin Risk Manag 2007;3(1):19-27.

2. Pratice guidelines for acute pain management in the perioparative setting: an updated reported by the American Society ok Anesthesilogists Task Force an Acute Pain Management. Anesthesiology 2004;100(6):1573-81.

3. Smith G, Power I. Audit and bridging the analgesic gap. Anaesthesia 1998;53(6):521-2.

4. Thompson JP, Bower S, Liddle AM, et al. Perioperative pharmacokinetics of transdermal fentanyl in elderly and young adult patients. Br J Anaesth 1998;81(2):152-4.

5. Gourlay GK. Treatment of cancer pain with transdermal fentanyl. Lancet Oncol 2001;2(3):165-72.

6 . Viscusi ER, Reynolds L, Tait S, et al. An iontophretic fentanyl patient-activated analgesic delivery system for postoperative pain: a double-blind, placebo-controlled trial. Anesth Analg 2006;102(2):188-94.

7. Summer GJ, Puntillo KA, Miaskowski C, et al. Burn injury pain: the continuing challenge. J Pain 2007;8(7):533-48.

8. Apfelbaum JL, Chen C, Mehta SS, et al. Postoperative pain experience: results from a national survey suggest postoperative pain continues to be undermanaged. Anesth Analg 2003;97(2):534-40.

9. Salomaki TE, Hokajarvi TM, Ranta P, et al. Improving the quality of postoperative pain relief. Eur J Pain 2000;4(4):367-72.

10. Dolin SJ, Cashman JN, Bland JM. Effectiveness of acute postoperative pain management: I. Evidence from published data. Br J Anaesth 2002;89(3):409-23. 11. Sathyan G, Jaskowiak, Evashenk M, et al. Characterisation of the pharmacokinetics of fentanyl $\mathrm{HCl}$ patient-controled transdermal system (PCTS): effect of current magnitude and multiple-day dosing, and comparison with IV fentanyl administration. Clin Pharmacokinet 2005;44(Suppl 1):7-15.

12. Ground S, Hall J, Spacek A, et al. Iontophoretic transdermal system using fentanyl compared with patient-controlled intravenous analgesia using morphine for postoperative pain management. Br J Anesth 2007;98(6):806-15.

13. Viscusi ER, Siccardi M, Damaraju CV, et al. The Safety and efficacy of fentanyl iontophoretic transdermal system compared with morphine intravenous pacient-controlled analgesia for postoperative pain 
management: an analysis of pooled data from three randomized, active-controlled clinical studies. Anesth Analg 2007;105(5):1428-36.

14. Chelly JE, Grass J, Houseman TW, et al. The safety and the efficacy of a fentanyl patient-controlled transdermal system for acute postoperative analgesia: a multicenter, placebo-controlled trial. Anesth Analg 2004;98(2):427-33.

15. Skinner HB. Multimodal acute pain management. Am J Orthop 2004;33(5 Suppl):5-9.

16. Closs SJ, Barr B, Briggs M, et al. A comparison of five pain assessment scales for nursing home residents with varying degrees of cognitive impairment. J Pain Symptom Manage 2004;27(3):196-205.

17. Minkowitz HS, Rathmell JP, Vallow S. et al. Efficacy and safety of the fentanyl iontophoretic transdermal system (ITS) and intravenous patient-controlled analgesia (IV PCA) with Morphine for pain mana- gement following abdominal or pelvic surgery. Pain Med 2007;8(8):657-68.

18. Pennington P, Caminiti S, Schein JR, et al. Patients' assessment of the convenience of fentanyl HCI iontophoretic transdermal system (ITS) versus morphine intravenous patient-controlled analgesia (IV PCA) in the management of postoperative pain after major surgery. Pain Manag Nurs 2009;10(3):124-33. 19. Panchal SJ, Damaraju CV, Nelson WW, et al. System-related events and analgesic gaps during postoperative pain management with the fentanyl iontophoretic transdermal system and morphine intravenous patient-controlled analgesia. Anesth Analg 2007;105(5):1437-41.

Apresentado em 28 de outubro de 2010.

Aceito para publicação em 02 de junho de 2011. 